\title{
Obstetrical view of COVID-19: a call for appropriate prenatal care during public health emergencies
}

Xiyao Liu ${ }^{1}$, Yuan Shi ${ }^{2}$, Miao Chen ${ }^{1}$, Jun Zhang ${ }^{3}$, Jianhui Wang ${ }^{2}$, Philip Baker ${ }^{4}$, Chao Tong $^{1}$, Xin $\mathrm{Luo}^{1}$, and Hongbo Qi ${ }^{1}$

${ }^{1}$ Chongqing Medical University First Affiliated Hospital

${ }^{2}$ Chongqing Medical University Affiliated Children's Hospital

${ }^{3}$ Shanghai Jiao Tong University School of Medicine

${ }^{4}$ University of Leicester

April 28, 2020

\section{Obstetrical view of COVID-19:}

a call for appropriate prenatal care during public health emergencies

Xiyao $\mathrm{Liu}^{1,2, \mathrm{a}}$, Yuan $\mathrm{Shi}^{3, \mathrm{a}}$, Miaomiao Chen ${ }^{1,2,4}$, Jun Zhang ${ }^{5}$, Jianhui Wang ${ }^{3}$, Philip N. Baker ${ }^{2,6}$, Chao Tong $^{1,2}$, Xin Luo ${ }^{1,2,}$, Hongbo $\mathrm{Qi}^{1,2, *}$

${ }^{1}$ Department of Obstetrics, The First Affiliated Hospital of Chongqing Medical University, No. 1 Youyi Road, Yuzhong District, Chongqing 400016, China.

${ }^{2}$ China-Canada-New Zealand Joint Laboratory of Maternal and Fetal Medicine, Chongqing Medical University, No.1 Yixueyuan Road, Yuzhong District, Chongqing 400016, China.

${ }^{3}$ Department of Neonatology, Children's Hospital of Chongqing Medical University, Chongqing 400014, China.

${ }^{4}$ Maternal and Child Health Hospital of Hubei Province, No. 745 Wuluo Road, Hongshan District, Wuhan City, Hubei Province, 430070, China.

${ }^{5}$ MOE-Shanghai Key Laboratory of Children's Environmental Health, Xin Hua Hospital Affiliated to Shanghai Jiao Tong University School of Medicine, Shanghai 200092, China.

${ }^{6}$ College of Life Sciences, University of Leicester, Leicester LE1 7RH, UK.

a Authors contributed equally.

* Correspondence to: Hongbo Qi (Address: Department of Obstetrics, The First Affiliated Hospital of Chongqing Medical University, No. 1 Youyi Road, Yuzhong District, Chongqing 400016, China. E-mail: qihongbocy@gmail.com. Telephone: +86 13808376116); Xin Luo (Address: Department of Obstetrics, The First Affiliated Hospital of Chongqing Medical University, No. 1 Youyi Road, Yuzhong District, Chongqing 400016, China. E-mail: lxcqmu@outlook.com. Telephone: +86 15826109160)

Running title: Appropriate Prenatal Care During COVID-19 and PHEs

Key words: SARS-CoV-2; COVID-19; prenatal care; public health emergencies

In late December 2019, an outbreak of a coronavirus disease, later termed COVID-19, emerged in China. ${ }^{1}$ Till March 31, 2020, this disease has affected over 690,000 people worldwide and caused more than 33,000 
deaths. ${ }^{2}$ WHO authorities alarmed an "very high level" of public health emergency of international concern (PHEIC). ${ }^{3}$

Pregnant women are vulnerable to this respiratory epidemic, because of their immunosuppressive state, and physiological adaptive changes (e.g., diaphragm elevation, increased oxygen consumption, and oedema of respiratory tract mucosa), which could cause hypoxia intolerance. ${ }^{4}$ As new evidences came to light, and based on lessons learnt from other similar diseases (SARS and MERS), China's National Health Commission issued a notice on February 8. This announcement claimed that pregnant women and new-borns should be regarded as a susceptible population to COVID-19, and called for the prevention and management strategies for them. ${ }^{5}$

At present, the limited reports on pregnant women mostly focus on their risks of infection, clinical characteristics, and the management of suspected infection. ${ }^{4,6,7}$ To the best of our knowledge, there are approximately more than 100 pregnant women with confirmed COVID-19 nationwide. In consideration of the fact that the annual number of births in China was much considerable, 14.65 million (2019) and 15.23 million (2018), ${ }^{8}$ we can modestly infer that the majority of pregnant women were not infected but affected. It also alarms us of the rationality of attention-shift to the regular care of pregnant women during this particular period. During this epidemic and other potential PHE henceforth, improving prenatal care to prevent evitable adverse pregnancy outcomes and to improve the quality of births became paramount. ${ }^{9}$

The impact of COVID-19 on prenatal status raised many obstetrical concerns. In response to the outbreak, all provinces in mainland China with confirmed cases of COVID-19 have adopted the first-level PHE responses since January 29, 2020. ${ }^{10}$ These measures include travel restrictions and executive orders on daily life. However, the restriction had its dual effects. Since the epidemic was gradually under control, the negative effects on psychological and physical aspects started to appear, especially for pregnant mothers, who are scheduled to make prenatal visits with obstetrical guidance.

Mental health is of great concern. Anxiety has been documented as a common psychological problem during pregnancy. ${ }^{11}$ Influenced by both accurate and erroneous news, pregnant mothers are suffering an excessive level of prenatal anxiety from carrying baby, epidemical threat, and strict restrictions. According to our preliminary data, the rate of prenatal anxiety during earlier period (from February $3^{\text {rd }}$ to $9^{\text {th }}$ ) was elevated to about $25 \%$ in the central area of this epidemic (Wuhan). The influence factors of this anxiety during this time was different from those in the non-PHE situation. Notably, prenatal anxiety was also demonstrated to alert long-term outcomes, such as postpartum depression. ${ }^{12}$

Crucially, physical health was inevitably affected. Firstly, home confinement reduced routine physical activities. IFG (impaired fasting glucose) and IGT (impaired glucose tolerance) due to undue weight gain have already been observed in clinical practice, which is of great concern to the obstetricians. Besides, the emergency traffic bans have made some medical resources inaccessible, and anxiety may deter women from attending routine prenatal care. ${ }^{13}$ Overall, the scheduled prenatal check and hospitalized delivery of pregnant women were postponed or cancelled. In our recent study, many pregnant women reported that they would rather miss vital check-ups than go to a hospital within one month. Although we have anticipated this phenomenon, the actual situation is still far beyond our cognition. In our hospitals, for example, about 6859 outpatient visits were completed to the First Affiliated Hospital of Chongqing Medical University is count in February 2020 (Figure 1), while in last February, it was 16120. This figure change in outpatient visits of pregnant mothers to Maternal and Child Health Hospital of Hubei Province was more unreasonable (Figure 2), and it was 5410 and 27254 during this and last February. Even if they came for the prenatal visit, exaggerated self-considered "protective measures" were commonly taken (Figure 3 and 4). The shortand long-term impacts of lack of prenatal care are of great concern. ${ }^{9}$

According to the Development Report on Chinese Maternal and Child Health (2019) ${ }^{14}$ in the past two decades, the rate of accessing prenatal care in China has steadily increased: overall, from $83.7 \%$ in 1996 to $96.6 \%$ in 2018; as for rural areas, from $80.6 \%$ to $95.8 \%$. Such improvement in prenatal care is closely related to the policy-making of the authorities and the prominent efforts from national obstetricians. The 
corresponding author, Professor Qi, has participant in the draft of Guideline of Preconception and Prenatal Care (2018) . ${ }^{15}$ Referring to the guidelines from the US, the UK, Canada, and WHO, the 2018 version of the guideline was under the basis of the Guideline of Preconception and Prenatal Care (2011), ${ }^{16}$ followed relevant Chinese laws and policies, and considered the requirements for Health Economics in China. The contents of this guideline include: health education and guidance, routine health care, and auxiliary examination items (including required items and optional items). Over the past few years, Chinese obstetricians have been working on the nationwide promotion of this guideline and calling for standardized prenatal care to reduce adverse pregnancy outcomes. However, on this unpredictable period of COVID-19 outbreak, an appropriate update of recommendations is needed urgently.

To standardize the management during the outbreak of COVID-19, the National Health Commission has already published seven versions of guidelines for diagnosis and treatment, and many institutions have also formulated their own management manuals. Under this context, our group has put forward a contingency plan for the management COVID-19 outbreak in NICUs. ${ }^{17}$ However, as for pregnant women, there is still a lack of evidence-based advice or strategies for prenatal care.

There are many undetermined problems for prenatal care during this period. Above all, the indications and strategies for the screening of SARS-CoV-2 among pregnant women varied in different institutions. For instance, in the epicentre, some hospitals perform a routine workup for all pregnant women waiting for delivery, including chest CT scan and virus detection test for SARS-CoV-2 (e.g., nucleic acid test from nasopharyngeal and oropharyngeal swab, antigen and antibody detection test). These sequacious screenings squandered medical resources and potentially aggravated the anxiety of pregnant women. Moreover, consequences of restrictions and lack of activities on prenatal health (e.g., GDM, macrosomia, excessive amniotic fluid, shoulder dystocia, and the increased CS rate) deserve attention. Additionally, the schedule changes of prenatal care caused by various reasons, and following potential adverse pregnancy outcomes, invoke immediate action. Last but not least, under this inconvenient situation, how to perform prenatal care and to relieve the workload of medical staff, is hotly discussed.

Here, we call for an appropriate contingency plan for prenatal care during the PHE to minimize the potential risk of adverse maternal and foetal outcomes.

The following recommendations we put forward were based on the existing experience:

Rational and standardized workflow for the screening of pregnant women

Screening strategies among pregnant women should be based on their risk of infection: ${ }^{18}$ Specific epidemiological history: o For low-risk pregnant women referred to hospital

Flowing prenatal examinations should not be postponed beyond its opportune gestational age: Ultrasound examination for Prenatal care out of hospital

Mental health is recommended to be included in perinatal care during PHE: Close cooperation of psychologists, obstetriciar

We have to be aware of the challenges and concerns brought by SARS-CoV-2 to our obstetrical field. We hope that these findings and suggestions can provide guidance and reference for the appropriate prenatal care in the epidemic areas worldwide.

\section{Funding}

National Natural Science Foundation of China (No. 81771614 and No. 81771613), and the National Key Research and Development Program of China (No. 2016YFC1000407).

\section{Ethics approval}

Not required.

\section{Contribution to Authorship}


This commentary was conceived by HBQ and XL. XYL and MMC prepared the first draft, and finalised the manuscript on the basis of JHW's proofreading, the comments, and reviewer feedback. JZ, YS, PB, and CT provided critical commentary from aspects of Public Health, Neonatology, Obstetrics and laboratory test. HBQ and XL revised the final version and are guarantors of this manuscript. All authors made substantial contributions to the paper and read and approved the final manuscript.

\section{Disclosure of Interests}

The author declares that he has no competing interests.

\section{Acknowledgments}

Here, we thank the Chinese Medical Association, Chapter of Perinatal Medicine, for their constant efforts on improving prenatal care by promoting the Guideline of Preconception and Prenatal Care. We also thank the nationwide obstetricians, nurses, and social workers who are providing care for the pregnant mothers during this difficult time.

\section{References}

1. Wuhan Municipal Health Commission. Report of clustering pneumonia of unknown aetiology in Wuhan City, 2019. http://wjw.wuhan.gov.cn/front/web/showDetail/2019123108989 (accessed Jan 20, 2020 [in Chinese]).

2. WHO. Coronavirus disease 2019 (COVID-19) situation report - 70. 30 March, 2020. https://www.who.int/docs/defaultsource/coronaviruse/situation-reports/20200330-sitrep-70-covid-19.pdf?sfvrsn=7e0fe3f8_2 (accessed 31 Mar, 2020).

3. WHO. WHO Director-General's opening remarks at the media briefing on COVID-19 - 28 February 2020. https://www.who.int/dg/speeches/detail/who-director-general-s-opening-remarks-at-the-mediabriefing-on-covid-19 - 28-february-2020 (accessed 3 Mar, 2020).

4. Chen H, Guo J, Wang C, et al. Clinical characteristics and intrauterine vertical transmission potential of COVID-19 infection in nine pregnant women: a retrospective review of medical records. The Lancet 2020. DOI: 10.1016/S0140-6736(20)30360-3

5. National Health Commission of the People's Republic of China. http://www.nhc.gov.cn/fys/s7902/202002/de2d62a5711c41 (accessed 12 Feb, 2020 [in Chinese]).

6. Qiao J. What are the risks of COVID-19 infection in pregnant women? The Lancet 2020. DOI: 10.1016/S01406736(20)30365-2

7. Favre G, Pomar L, Qi X, Nielsen-Saines K, Musso D, Baud D. Guidelines for pregnant women with suspected SARS-CoV-2 infection. Lancet Infect Dis 2020. DOI: 10.1016/S1473-3099(20)30157-2

8. National Bureau of Statistics of the People's Republic of China. http://www.stats.gov.cn/tjsj/sjjd/202001/t20200117_1723470.html (accessed 5 Mar, 2020 [in Chinese]).

9. Dowswell T, Carroli G, Duley L, et al. Alternative versus standard packages of antenatal care for low-risk pregnancy. Cochrane Database Syst Rev 2015; (7): CD000934.

10. China Daily. Tibet activates highest-level public health alert. Jan 29, 2020. https://www.chinadaily.com.cn/a/202001/29 (accessed 4 Feb, 2020).

11. Yan W, Wang X, Kuang H, et al. Physical activity and blood pressure during pregnancy: Mediation by anxiety symptoms. J Affect Disord2020; $264: 376-82$.

12. Milgrom J, Gemmill AW, Bilszta JL, et al. Antenatal risk factors for postnatal depression: a large prospective study. J Affect Disord2008; 108 (1-2): 147-57. 
13. Cheng BH, Chen JH, Wang GH. Psychological factors influencing choice of prenatal diagnosis in Chinese multiparous women with advanced maternal age. J Matern Fetal Neonatal Med 2019; 32 (14): 2295-301.

14. National Health Commission of the People's Republic of China. Development Report on Chinese Maternal and Child Health (2019). http://www.nhc.gov.cn/fys/s7901/201905/bbd8e2134a7e47958c5c9ef032e1dfa2.shtml (accessed 5 Mar, 2020 [in Chinese]).

15. Obstetrics Subgroup, Chinese Society of Obstetrics and Gynecology, Chinese Medical Association. Guideline of preconception and prenatal care (2018). Chin J Obstet Gynecol 2018; 53 (1): 7-13.(in Chinese)

16. Obstetrics Subgroup, Chinese Society of Obstetrics and Gynecology, Chinese Medical Association. Guideline of preconception and prenatal care (1st edition). Chin J Obstet Gynecol 2011; 46 (2). (in Chinese)

17. Wang J, Qi H, Bao L, Li F, Shi Y. A contingency plan for the management of the 2019 novel coronavirus outbreak in neonatal intensive care units. Lancet Child Adolesc Health 2020. DOI: 10.1016/S2352$4642(20) 30040-7$

18. National Health Commission of the People's Republic of China. Prevention and control of COVID-19 (6th edition). 7 March, 2020. http://www.nhc.gov.cn/jkj/s3577/202003/4856d5b0458141fa9f376853224d41d7/files/4132bf035bc2 (accessed 7 Mar, 2020 [in Chinese]).

19. WHO, United Nations Population Fund, UNICEF. Pregnancy, childbirth, postpartum and newborn care: A guide for essential practice (3rd edition). https://apps.who.int/iris/bitstream/handle/10665/249580/9789241549356eng.pdf;jsessionid=586E220FF9B5DBFF2DA34C7896836883?sequence=1 (accessed 11 Feb, 2020). 


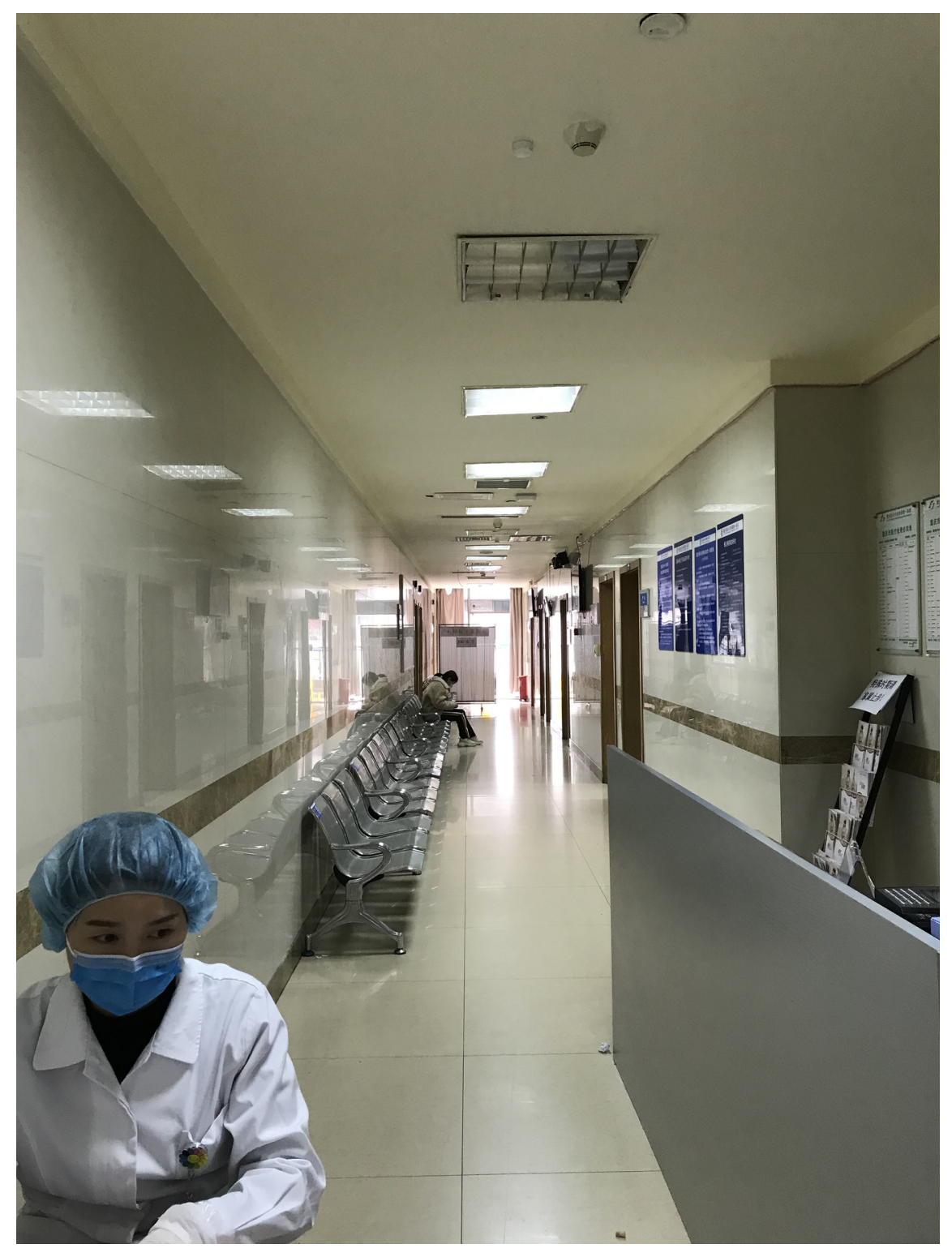




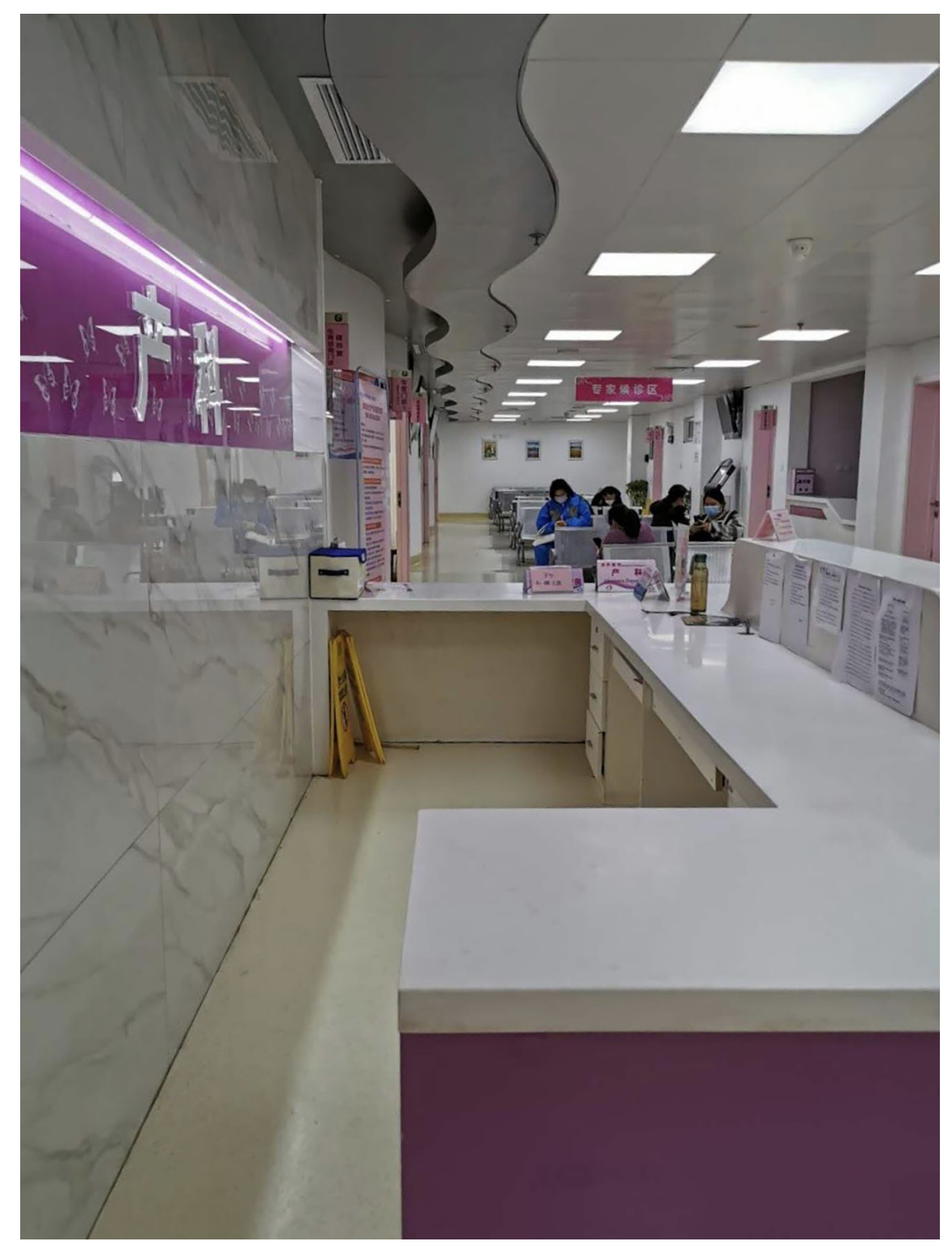




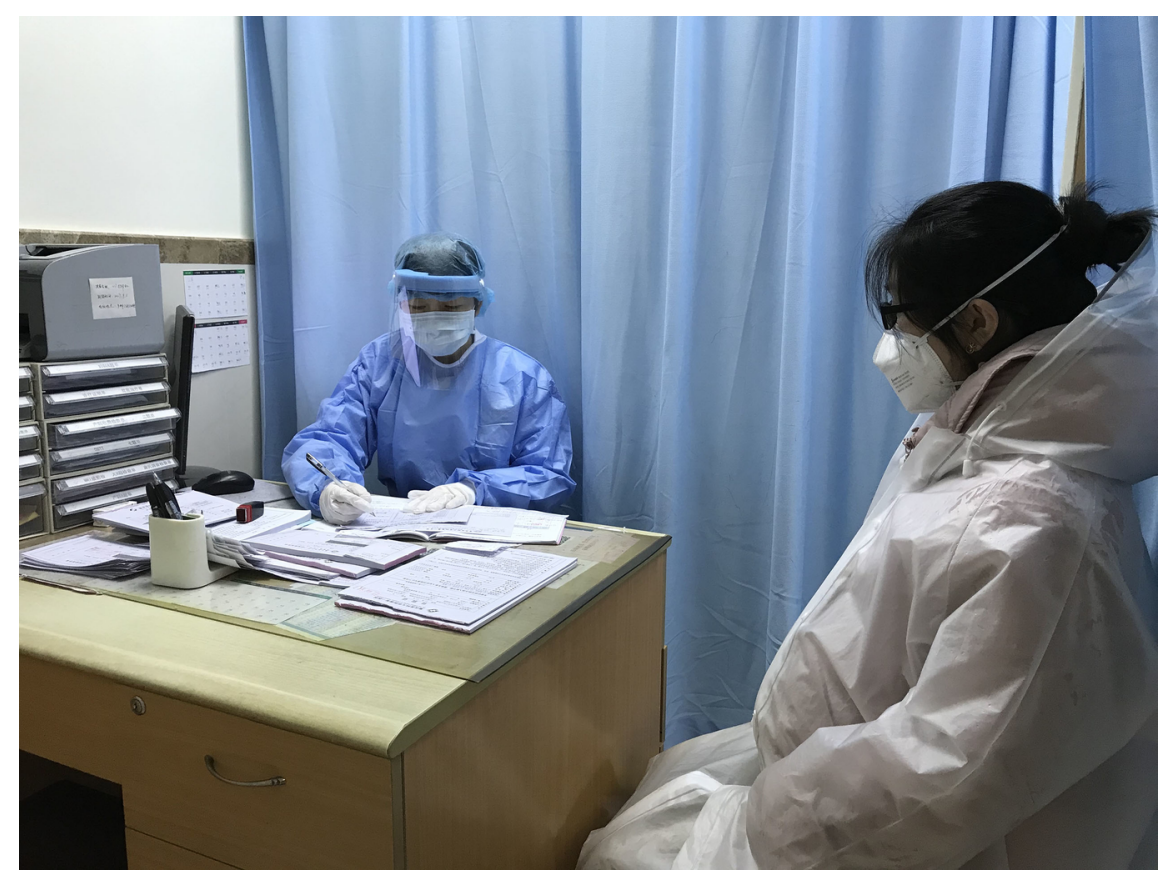




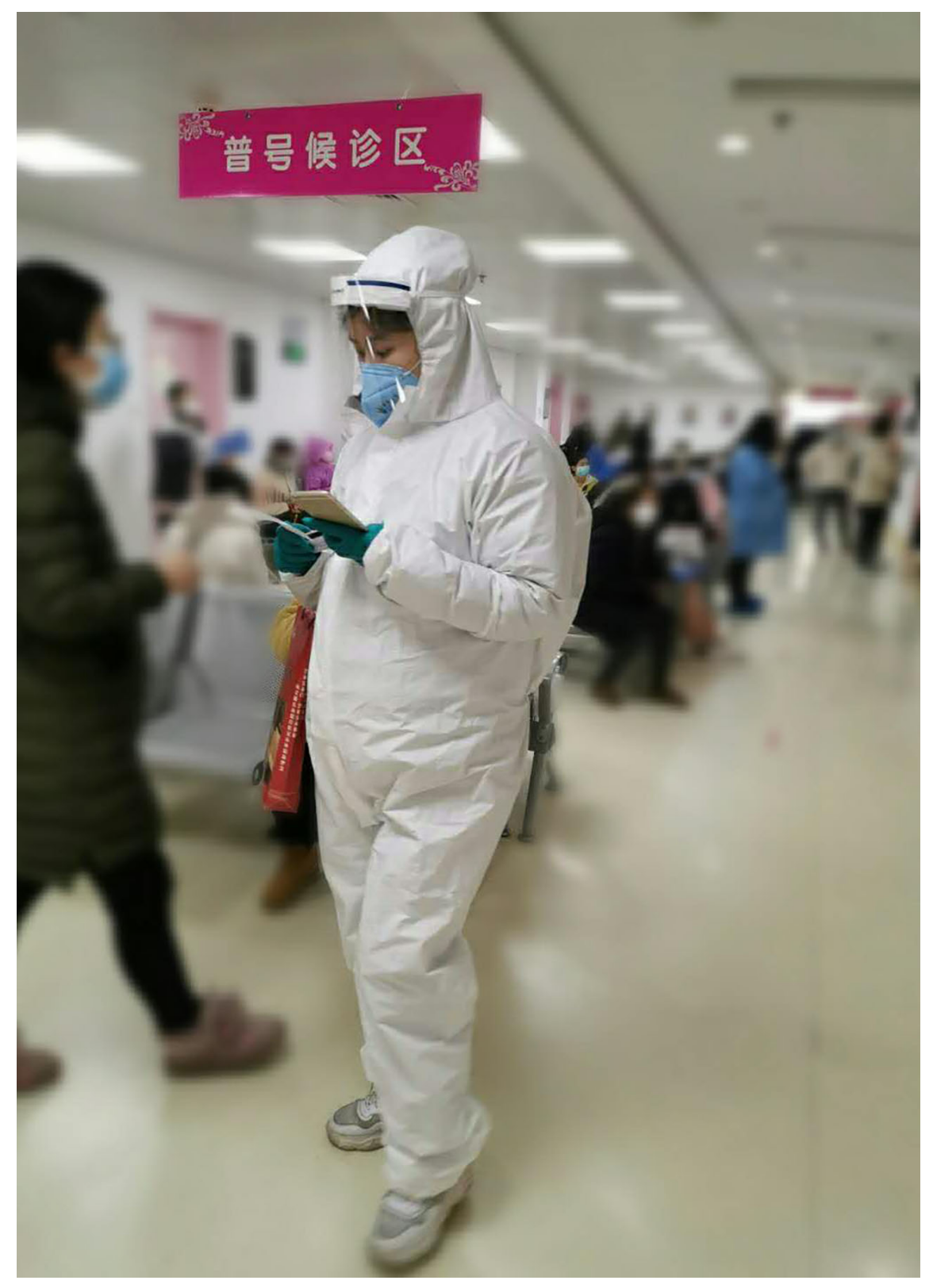

